\title{
IRAS MINOR PLANET SURVEY
}

\author{
EDWARD F. TEDESCO \\ Mission Research Corporation, One Tara Boulevard, Nashua, NH 03062 USA \\ E-mail etedesco@dirac.plh.af.mil
}

\begin{abstract}
This chapter describes the contents and organization of the IRAS Minor Planet Survey Data Base and tells how to obtain hard-copy and machine-readable versions of the data.

The primary purpose of the Infrared Astronomical Satellite (IRAS) was to survey the sky in four wavelength bands centered near 12, 25, 60 and $100 \mu \mathrm{m}$. The satellite was launched in January 1983 and obtained observations until November 1983. In this period it surveyed approximately 96 percent of the sky. The IRAS mission, data processing and data products are described in a series of catalogs. These catalogs all deal with sources which are fixed on the sky. The IRAS Asteroid and Comet Survey, 1986 provided observations for moving sources, the asteroids and comets.

This chapter summarizes the creation of the second IRAS catalog for moving sources, the IRAS Minor Planet Survey (IMPS). It describes the contents and organization of this catalog and tells how to obtain hard-copy and machine-readable versions of the data.
\end{abstract}

\section{The IRAS Minor Planet Survey}

In contrast with the IRAS Asteroid and Comet Survey (1986), IMPS processed only IRAS survey observations of asteroids; comets were not processed. Low Resolution Spectrometer, Serendipitous, and Additional Observations data were not processed. IMPS did process all asteroids with reasonably-well-known orbits as of December 1990. In particular, IMPS updates the processing of asteroids numbered 1 through 3318 and extends this processing to asteroid number 4679 plus 2,632 asteroids with preliminary (two or more opposition) orbits.

Note that, as with the IRAS Asteroid and Comet Survey (1986), the IMPS catalogs and databases are fundamentally different from those catalogs and databases produced for fixed sources. Asteroids move and their apparent emission levels can vary by large amounts. Consequently different methods and criteria were used for processing potential asteroid sightings. The IMPS documentation describes these differences.

\section{The IRAS Minor Planet Survey Catalog}

The IRAS Minor Planet Survey (1992) consists of a 460 page hard-copy document. This document presents a history of the IRAS asteroid task, identifies the ground-based asteroid data used in associating asteroids with IRAS sources and in deducing albedos and diameters from their IRAS-measured infrared fluxes, and describes how asteroid sightings were identified and processed. It presents analyses of the asteroid associations and accepted sightings, an overview of the results obtained. The descriptive part concludes with a summary chapter which discusses 
the completeness and reliability of the survey, the statistical adjustments made to the derived results, and details the major differences between this catalog and the IRAS Asteroid and Comet Survey (1986).

Following the descriptive section the document describes the IMPS data products and presents all of the IRAS Minor Planet Survey catalogs, i.e., all of the printed data products. This section contains technical details about the data, including data formats. It presents a general description of the final data products, gives the formats of the data bases and catalogs, presents a sub-set of the ground-based data used (the corresponding data base contains all the ground-based data used), and presents albedos and diameters derived for all asteroids with multiple sightings and, separately, for those asteroids with only a single IRAS sighting at one wavelength. Additional products present data on detection statistics, rejected sightings, and missed-predictions. Also included are a table of acronyms and glossary of terms, and the IRAS flux look-up table (used to convert an IRAS flux into a visual geometric albedo).

\section{Final Data Products}

The printed data products are referred to as "catalogs" and the machine-readable products as "data bases". All of the catalogs appear in the hard-copy document; the machine-readable data bases are available via ftp from the National Space Science Data Center (NSSDC) at the Goddard Space Flight Center under the name The IRAS Minor Planet Survey Catalog and Data Base, 1992.

The machine-readable data (Tedesco, et al., 1992) may be obtained from the National Space Science Data Center by sending an electronic mail message to :

\section{REQUEST@NSSDCA.GSFC.NASA.GOV (Internet) or to :}

\section{NSSDCA : : REQUEST (NSI-DECnet).}

Arrangements to obtain them may also be made via telephone at (301) 2866695 or by writing to : NSSDC Coordinated Request and User Support Office, NASA/Goddard Space Flight Center, Code 633.4, Greenbelt, MD 20771 U.S.A.

The hard-copy document [IRAS Minor Planet Survey (1992)] is only available from Dr. Stephan Price, Phillips Laboratory, Geophysical Directorate, Backgrounds Branch/GPOB, Hanscom Air Force Base, MA 01731-3010 (Internet : price(@)dirac.plh.af.mil). Furthermore, the only supported version of the machinereadable data base is that at the NSSDC.

\section{Catalogs and Data Bases}

Table 1 presents a concise summary of the available data products. With the exception of a portion of the IMPS Ground-Based Data Catalog (FP 107) and the entire IMPS Sightings Data Base (FP 108) all products exist as both a "Catalog", i.e., as tables appearing in the hard-copy documentation, and as a "Data Base", i.e., as ASCII data file(s). 
TABLE I

IMPS DATA PRODUCTS

\begin{tabular}{|c|c|c|}
\hline $\begin{array}{l}\text { Final } \\
\text { Product No. } \\
\text { (Size in Kb) }\end{array}$ & Final Product Name & Remarks \\
\hline $\begin{array}{r}100 \\
(\mathrm{NA}) \\
\end{array}$ & $\begin{array}{l}\text { The IRAS Minor Planet } \\
\text { Survey }\end{array}$ & Hard-copy documentation. \\
\hline $\begin{array}{r}101 \\
(20) \\
\end{array}$ & $\begin{array}{l}\text { IMPS Final Products } \\
\text { Format Catalog }\end{array}$ & $\begin{array}{l}\text { This catalog gives the formats of all } \\
\text { the machine-readable data products. }\end{array}$ \\
\hline $\begin{array}{c}102 \\
(239)\end{array}$ & $\begin{array}{l}\text { IMPS Albedos and } \\
\text { Diameters Catalog }\end{array}$ & $\begin{array}{l}\text { A distilled summary of the averaged } \\
\text { results for the } 1,796 \text { numbered } \\
\text { asteroids and } 88 \text { unnumbered } \\
\text { asteroids with at least two accepted } \\
\text { observations. }\end{array}$ \\
\hline $\begin{array}{l}103 \\
(15)\end{array}$ & IMPS Singleton Catalog & $\begin{array}{l}\text { Same as Final Product } 102 \text { but for the } \\
94 \text { numbered and } 26 \text { unnumbered } \\
\text { asteroids which have only a single } \\
\text { accepted sighting in a single band. }\end{array}$ \\
\hline $\begin{array}{c}104 \\
(193)\end{array}$ & IMPS Statistics Catalog & $\begin{array}{l}\text { A summary of the number of times } \\
\text { each asteroid was sighted, the } \\
\text { number of times it was predicted to be } \\
\text { scanned, and possible reasons for } \\
\text { any failure to be detected. }\end{array}$ \\
\hline $\begin{array}{c}105 \\
(100)\end{array}$ & IMPS Reject Catalog & $\begin{array}{l}\text { A summary of the number of rejected } \\
\text { sightings for each asteroid and } \\
\text { possible reasons for their rejection. }\end{array}$ \\
\hline $\begin{array}{c}106 \\
(320)\end{array}$ & $\begin{array}{l}\text { IMPS Missed-Predictions } \\
\text { Catalog }\end{array}$ & $\begin{array}{l}\text { A summary of asteroids which were } \\
\text { scanned by the IRAS focal plane array } \\
\text { but which did not generate any } \\
\text { associations. }\end{array}$ \\
\hline $\begin{array}{c}107 \\
(2,385) \\
\end{array}$ & $\begin{array}{c}\text { IMPS Ground-Based Data } \\
\text { Catalog }\end{array}$ & $\begin{array}{l}\text { A listing of ground-based data used in } \\
\text { IMPS data processing. }\end{array}$ \\
\hline $\begin{array}{c}108 \\
(4,538)\end{array}$ & IMPS Sightings Data Base & $\begin{array}{l}\text { A listing of } 7,937 \text { accepted sightings } \\
\text { associated with } 1,890 \text { numbered } \\
\text { asteroids and } 273 \text { accepted sightings } \\
\text { associated with } 114 \text { unnumbered } \\
\text { asteroids. }\end{array}$ \\
\hline
\end{tabular}


For the most part, the formats of the Catalog and Data Base versions of each data product are the same. In some cases, however, there are differences introduced to improve the readability of the Catalog version. These differences are noted in the appropriate places.

The hard-copy documentation, together with the machine-readable files of the final data products, constitute The IRAS Minor Planet Survey Catalog and Database, 1992. This supplants the 1986 version IRAS Asteroid and Comet Database and Catalog. A cross reference between final data products from the IRAS Asteroid and Comet Database and Catalog, 1986 and The IRAS Minor Planet Survey Catalog and Database, 1992 is given in the hard-copy documentation.

\section{Acknowledgements}

Creation of the IMPS catalogs and data bases was made possible through grants from the Phillips Laboratory, Geophysical Directorate under funding provided by the Strategic Defense Initiative Organization.

\section{References}

Infrared Astronomical Satellite Asteroid and Comet Survey : Preprint Version No. 1, 1986, edited by Matson, D.L., JPL Internal Document No. D- 3698. (Jet Propulsion Laboratory, Pasadena)

IRAS Minor Planet Survey, 1992, edited by Tedesco, E.F. (Phillips Laboratory Technical Report No. PL-TR-92-2049. Hanscom Air Force Base, MA.)

Tedesco, E.F., Veeder, G.J., Fowler, J.W., and Chillemi, J.R (1992). The IRAS Minor Planet Survey Data Base, National Space Science Data Center, Greenbelt, MD. 